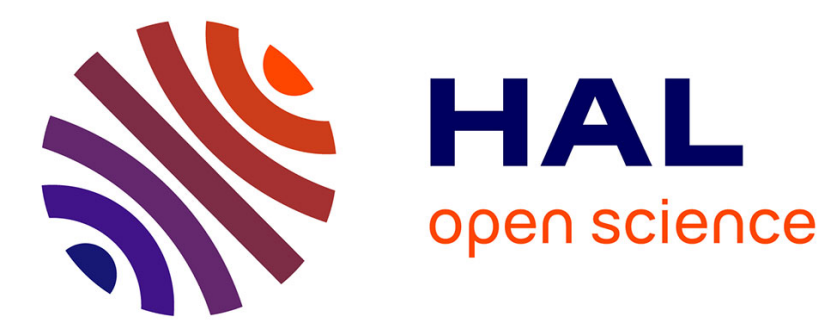

\title{
X-ray photochemistry of carbon hydride molecular ions
}

Alessandra Puglisi, Tsveta Miteva, Eugene T Kennedy, Jean-Paul Mosnier, Jean-Marc Bizau, Denis Cubaynes, Nicolas Sisourat, Stéphane Carniato

\section{To cite this version:}

Alessandra Puglisi, Tsveta Miteva, Eugene T Kennedy, Jean-Paul Mosnier, Jean-Marc Bizau, et al.. X-ray photochemistry of carbon hydride molecular ions. Physical Chemistry Chemical Physics, 2018, 20 (6), pp.4415-4421. 10.1039/C7CP08026C . hal-01723042

\section{HAL Id: hal-01723042 \\ https://hal.sorbonne-universite.fr/hal-01723042}

Submitted on 5 Mar 2018

HAL is a multi-disciplinary open access archive for the deposit and dissemination of scientific research documents, whether they are published or not. The documents may come from teaching and research institutions in France or abroad, or from public or private research centers.
L'archive ouverte pluridisciplinaire HAL, est destinée au dépôt et à la diffusion de documents scientifiques de niveau recherche, publiés ou non, émanant des établissements d'enseignement et de recherche français ou étrangers, des laboratoires publics ou privés. 
Cite this: DOI: $10.1039 / x x x x x x x x x x$

Received Date

Accepted Date

DOI: $10.1039 / x x x x x x x x x x$

www.rsc.org/journalname

\section{X-ray photochemistry of carbon hydride molecular ions}

\author{
Alessandra Puglisi, ${ }^{a}$ Tsveta Miteva, ${ }^{a}$ Eugene T. Kennedy, ${ }^{b}$ Jean-Paul Mosnier, ${ }^{b}$ Jean- \\ Marc Bizau ${ }^{c}$, Denis Cubaynes, ${ }^{c}$ Nicolas Sisourat, ${ }^{* a}$ and Stéphane Carniato ${ }^{a}$
}

Hydride molecular ions are key ingredients of the interstellar chemistry since they are precursors of more complex molecules. In regions located near a soft x-ray source these ions may resonantly absorb an x-ray photon which triggers a complex chain of reactions. In this work, we simulate $a b$ initio the x-ray absorption spectrum, Auger decay processes and the subsequent fragmentation dynamics of two hydride molecular ions, namely $\mathrm{CH}_{2}^{+}$and $\mathrm{CH}_{3}^{+}$. We show that these ions feature strong $x$-ray absorption resonances which relax through Auger decay within $7 \mathrm{fs}$. The doublycharged ions thus formed mostly dissociate into smaller ionic carbon fragments: in the case of $\mathrm{CH}_{2}^{+}$, the dominant products are either $\mathrm{C}^{+} / \mathrm{H}^{+} / \mathrm{H}$ or $\mathrm{CH}^{+} / \mathrm{H}^{+}$. For $\mathrm{CH}_{3}^{+}$, the system breaks primary into $\mathrm{CH}_{2}^{+}$and $\mathrm{H}^{+}$, which provides a new route to form $\mathrm{CH}_{2}^{+}$near a x-ray source. Furthermore, our simulations provide the branching ratios of the final products formed after the $x$-ray absorption as well as their kinetic and internal energy distributions. Such data can be used in the chemistry models of the interstellar medium.

\footnotetext{
a Sorbonne Universités, UPMC Univ Paris 06, CNRS, Laboratoire de Chimie Physique Matière et Rayonnement, F-75005, Paris, France; E-mail: nicolas.sisourat@upmc.fr

${ }^{b}$ School of Physical Sciences and NCPST, Dublin City University, Dublin 9, Ireland

${ }^{c}$ Institut des Sciences Moléculaires d'Orsay, CNRS, Université Paris-Sud, and Université Paris-Saclay, F-91405 Orsay, France

d Synchrotron SOLEIL, L'Orme des Merisiers, Saint-Aubin, BP 48, F-91192 Gif-sur-Yvette Cedex, France

* email corresponding author: nicolas.sisourat@upmc.fr
} 


\section{Introduction}

Carbon hydride molecular ions $\left(\mathrm{CH}_{n}^{+}\right)$are key molecular species in the chemistry of the interstellar medium (ISM), where they play an important role in the formation of more complex molecules ${ }^{1,2}$. Atomic carbon is mostly present in the ISM in the charged state $\mathrm{C}^{+}$. This cation can react with $\mathrm{H}_{2}$ to form $\mathrm{CH}_{2}^{+}$through radiative association. $\mathrm{CH}_{2}^{+}$can further react with another $\mathrm{H}_{2}$ molecule whereupon $\mathrm{CH}_{3}^{+}$is produced. These two carbon hydride molecular ions are then intermediate species in forming, for example, $\mathrm{CO}$ or longer hydrocarbon molecules (see Fig. 1).

Owing to the importance of these two molecular ions, several uni- and bi-molecular reactions involving these species have been investigated (see the databases ${ }^{3-5}$ ). Among the former class of reactions, the interaction of these ions with ultraviolet (UV) radiation is important ${ }^{6}$ and provides other routes in the chemical networks describing the molecular evolution of interstellar clouds. It should be noted that most photons in the ISM have energies below $13.6 \mathrm{eV}^{7}$ and therefore the latter class of reactions are generally the most relevant processes.

However, x-ray photons are also present in some regions (see ${ }^{8}$ for a catalog of x-ray sources in astrophysical environments). To our knowledge, interaction of these molecular ions with high energy photons has not been considered so far. In the presence of an x-ray source, core electrons of carbon can be resonantly excited. Highly-excited species are thus formed which de-excite dominantly by (resonant) Auger decay (i.e. by ejection of an energetic electron). After the Auger process, the molecular ions are multiply-charged and thus generally dissociate in several charged fragments. The fragmentation pathways depend on the states populated in the resonant Auger decay.

In this paper, we investigate these unexplored routes (see Fig. 1) in order to provide a complete description of the carbon chemistry in the ISM. Among the carbon hydride molecular ions, X-ray absorption spectroscopy of $\mathrm{CH}^{+}$has been reported in ${ }^{9}$. Furthermore, it was shown that the $\mathrm{CH}^{2+}$ ions formed after Auger decay dissociate dominantly into $\mathrm{C}^{2+}$ and $\mathrm{H}$. X-ray induced chemistry of $\mathrm{CH}^{+}$is therefore well understood. Here, we focus specifically on the processes triggered by absorption of an x-ray photon in $\mathrm{CH}_{2}^{+}$and $\mathrm{CH}_{3}^{+}$molecular ions. The x-ray absorption spectra and Auger partial widths were computed in order to obtain the population of the doubly-charged ions which further dissociate. We then simulated the dissociation dynamics of these dications and we obtained the products formed as well as their kinetic and internal energies.

The outline of the article is the following: in section II, we describe the theoretical methods and computational details employed to calculate the x-ray absorption spectra, the Auger processes and subsequent fragmentation dynamics. In section III we present and discuss the results for the two molecular ions $\mathrm{CH}_{2}^{+}$and $\mathrm{CH}_{3}^{+}$. The article ends with the conclusions of this work. Atomic units are used throughout unless stated otherwise.

\section{Methods and computational details}

\section{X-ray absorption spectra}

The soft x-ray absorption spectra of $\mathrm{CH}_{2}^{+}$and $\mathrm{CH}_{3}^{+}$were calculated using the linear coupling model ${ }^{10}$. In this model the photoabsorption cross section $(\sigma)$ is given by

$$
\begin{gathered}
\sigma(\omega)=\frac{1}{3} \sum_{m}^{\text {bound }} \mu_{m}^{2} \sum_{v_{m}} f_{v_{m}} \frac{\Gamma}{\pi\left[\left(\omega-\left(E_{m}+e_{v_{m}}\right)\right)^{2}+\Gamma^{2} / 4\right]} \\
+\frac{1}{3} \sum_{m}^{\text {diss. }} \mu_{m}^{2} \int d E \frac{e^{-(E-\omega)^{2} / 2 \varepsilon_{m}}}{\varepsilon_{m} \sqrt{2 \pi}} \frac{\Gamma}{\pi\left[\left(\omega-E_{m}\right)^{2}+\Gamma^{2} / 4\right]}
\end{gathered}
$$

where $\omega$ is the photon energy, $\mu_{m}$ is the transition dipole moment between the ground state and the $\mathrm{m}^{\text {th }}$ core-excited state, $E_{m}$ is the vertical energy of the $\mathrm{m}^{\text {th }}$ core-excited state with respect to the ground state, $\Gamma$ is the core-excited width. The first term on the r.h.s. is a sum over all states having a bound potential energy surface. The inner sum is over all 


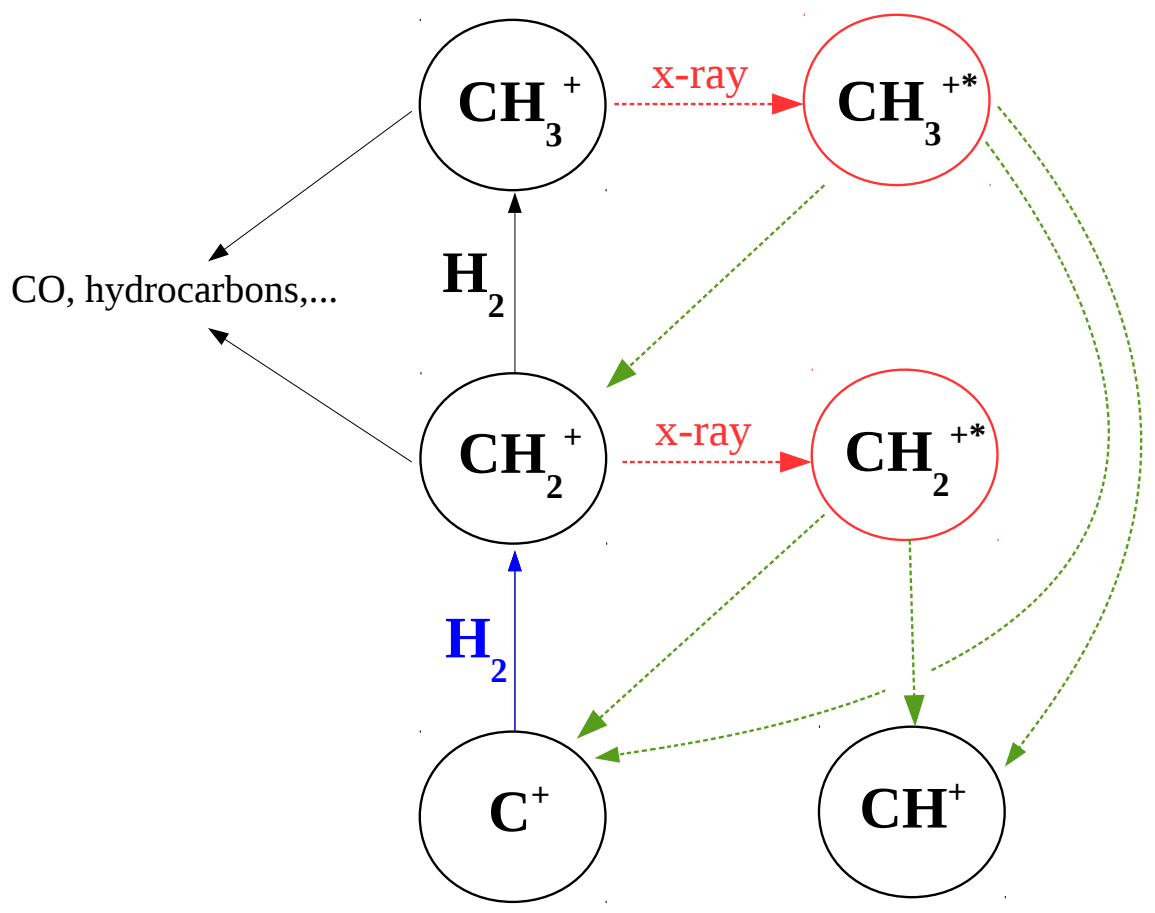

Fig. 1 Key reactions involving carbon hydride ions (full lines) and reactions investigated in this work (dashed lines). The blue line corresponds to radiative association, black lines are associated with ion-molecule reactions. Red lines indicate absorption of a soft $\mathrm{x}$-ray photon and green lines represent Auger-induced fragmentation pathways.

vibrational levels $v_{m}$ of the corresponding core-excited state. The Franck-Condon factors are denoted as $f_{v_{m}}$ and the vibrational energy level is $e_{v_{m}}$. The Franck-Condon factor $f_{v_{m}}$ corresponding to the transition from $(0,0, \ldots)$ towards $\left(v_{m}^{1}\right.$, $\left.v_{m}^{2}, \ldots\right)$ is obtained as follows

$$
f_{v_{m}}=\prod_{a} \frac{S_{a}^{v_{m}^{a}}}{v_{m}^{a} !} e^{-S_{a}}
$$

where $S_{a}=\frac{\omega_{a} \delta q_{a}^{2}}{2}$ and $\delta q_{a}$ is the change in the mass-weighted normal coordinate $q_{a}$ between the ground and core-excited states. The second sum runs over all states having dissociative character. The standard deviation $\varepsilon_{m}$ in this term is given by

$$
\varepsilon_{m}=\frac{1}{\sqrt{\omega_{a}}} \frac{\partial E_{m}}{\partial q_{a}}
$$

where $\omega_{a}$ is the corresponding frequency of the normal coordinate $q_{a}$. The spectra were convolved with a Gaussian function of FWHM of $0.28 \mathrm{eV}$ to include typical experimental resolution ${ }^{9}$.

The energies and transition dipole moments necessary for computing the cross section (Eq. (1)) were calculated with the post-Hartree-Fock Configuration Interaction (CI) method including single excitations, as implemented in the GAMESS-US quantum chemistry package ${ }^{11}$. This method has proven to provide spectra in quantitative agreement with experimental results on several molecular hydride ions ${ }^{12}$. The vertical energy and transition dipole moments 
were obtained with the aug-cc-pvQz basis set ${ }^{13}$ augmented with $7 \mathrm{~s}, 6 \mathrm{p}, 5 \mathrm{~d}$ and $3 f$ diffuse functions to ensure a better description of the excited states in the region below the ionization threshold. In order to take into account the electronic relaxation upon core-excitation we used Hartree-Fock molecular orbitals optimized for the lowest core-excited state of each species (see ${ }^{12}$ for details).

\section{Auger decay}

The total carbon K-shell Auger widths of several molecules have been determined by total ion yield and X-ray photoelectron spectroscopy (XPS) measurements ${ }^{14}$. It was shown that unless the carbon is attached to strong electronegative elements, like fluorine atoms, the total width weakly depends on the environment of the carbon. However, partial Auger widths depend on the molecule investigated. The number and character of the states reached after Auger decay are therefore functions of the molecule.

In this work we used the Fano-Configuration Interaction (Fano-CI) method ${ }^{15}$ to compute the total and partial widths of $\mathrm{CH}_{2}^{+}$and $\mathrm{CH}_{3}^{+}$molecular ions. The method was presented in detail in ${ }^{15}$. We briefly outline it here.

In the Fano theory of resonances ${ }^{16,17}$, the electronic wavefunction is a superposition of a discrete $\Phi$, and continuum $\chi_{\beta, \varepsilon}$ components. The Auger widths are then given by

$$
\Gamma=\sum_{\beta} \Gamma_{\beta}=2 \pi \sum_{\beta}\left|\left\langle\Phi\left|\hat{H}-E_{r}\right| \chi_{\beta, \varepsilon_{\beta}}\right\rangle\right|^{2}
$$

where $\Gamma$ is the total width and $\Gamma_{\beta}$ the partial width corresponding to the final state $\beta$. The kinetic energy of the Auger electron is $\varepsilon_{\beta}$ and $E_{r}$ is the energy of the core-excited state. In our implementation, the discrete part is a single Slater determinant corresponding to the excitation of a $1 \mathrm{~s}$ electron of carbon to the lowest unoccupied or singly occupied orbital. The continuum states are obtained by performing CI calculations where the Auger electron is approximated by the Hartree-Fock virtual orbitals. Since the latter do not have the proper boundary conditions, a Stieltjes imaging technique is employed to recover the correct widths from the approximated ones ${ }^{18-20}$.

As for the x-ray spectra, we used Hartree-Fock molecular orbitals optimized for the lowest core-excited state of each ion. The aug-cc-pVTZ basis set ${ }^{13}$ augmented with $7 \mathrm{~s}, 7 \mathrm{p}$ and $7 \mathrm{~d}$ even-tempered ${ }^{21}$ functions was employed. Convergence of the results with respect to the basis set was checked.

\section{Fragmentation dynamics}

In the Auger decay process following the core excitation of the molecular ions, a manifold of doubly ionized states is populated. In the course of the subsequent fragmentation dynamics the ions can go from one state to another owing to non-adiabatic couplings between the latter. In order to simulate this complex electronic and nuclear dynamics, we used the fewest switching surface hopping method as implemented in the SHARC package ${ }^{22,23}$.

In brief, classical trajectories are started on one of the potential energy surfaces. Along the propagation, the trajectories are allowed to hop from one surface to another according to the fewest switching criterion. Since the Auger decay takes place within a few femtoseconds, the nuclear dynamics before the decay are neglected and the initial conditions (positions and velocities of the nuclei) are taken from a quantum-harmonic Wigner distribution of the ground electronic state. Furthermore, the relative population of each doubly ionized state is obtained from the ratio of the partial and total Auger widths.

The gradients and the non-adiabatic couplings needed for the nuclear dynamics calculations are obtained with the MOLPRO package ${ }^{24,25}$. The potential energy surfaces were computed at the CASSCF level using the 6-31G** basis set ${ }^{26}$ and including the minimum number of active orbitals and all valence electrons. 


\section{Results and discussion}

\section{$\mathrm{CH}_{2}^{+}$}

The ground state equilibrium geometry of $\mathrm{CH}_{2}^{+}$has a $\mathrm{C}_{2 v}$ symmetry and its electronic configuration is $\left(1 a_{1}\right)^{2}\left(2 a_{1}\right)^{2}\left(1 b_{2}\right)^{2}\left(3 a_{1}\right)^{1}$. Note that the $1 a_{1}$ orbital corresponds to the 1 s orbital of carbon.

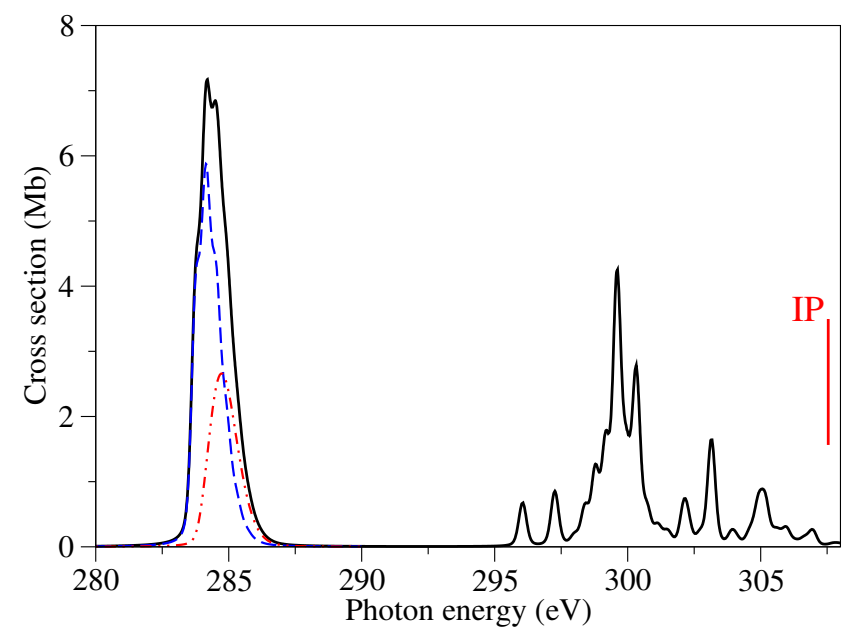

Fig. 2 Absolute $x$-ray photoabsorption cross sections of $\mathrm{CH}_{2}^{+}$, calculated with the linear coupling model (solid black line, see Eq. 1). The spectra corresponding to the $1 a_{1} \rightarrow 1 b_{1}$ and to the $1 a_{1} \rightarrow 3 a_{1}$ transitions are shown in blue dashed line and red dashed dotted line, respectively.

The absolute x-ray absorption spectrum of $\mathrm{CH}_{2}^{+}$is shown in Fig. 2. Furthermore, we computed the core-shell ionization threshold at the DFT/B3LYP level and found it at $307.5 \mathrm{eV}$ as indicated in the figure. The lowest and most intense band, centered below $285 \mathrm{eV}$, corresponds to two transitions: the excitation of a 1s electron of carbon to the lowest unoccupied molecular orbital (LUMO, i.e. the orbital $1 b_{1}$ ) and to the highest occupied molecular orbital (HOMO, i.e. $3 a_{1}$ ) which is singly occupied in the electronic ground state of $\mathrm{CH}_{2}^{+}$. The spectrum for the lowest transition exhibits a vibrational progression, which is not well resolved. The vertical energy of both transitions differs by about $100 \mathrm{meV}$ and the oscillator strengths of these transitions are about 0.1. For comparison, these transitions are between one and three orders of magnitude stronger than the transitions between the valence states of $\mathrm{CH}_{2}^{+}$(i.e. induced by UV photons) ${ }^{27}$. The higher lying peaks are more than $16 \mathrm{eV}$ higher in energy than the first one and exhibit smaller cross sections. In the following we consider only the two lowest transitions (i.e. below $285 \mathrm{eV}$ ) since they are the most intense ones. Furthermore, an accurate description of the Auger process and the subsequent fragmentations for the high-lying coreexcited states is not possible within the current implementation of our methods.

The lifetimes of the two lowest core-excited states computed using the Fano-CI method are $7.4 \mathrm{fs}$ which is in excellent agreement with the lifetime of the carbon core-ionized state measured for $\mathrm{CH}_{4}{ }^{14}$. The branching ratios for the different final states are shown in Table 1 for the $1 a_{1} \rightarrow 1 b_{1}$ transition and in Table 2 for the $1 a_{1} \rightarrow 3 a_{1}$ transition. The final states are labeled according to their spectroscopic term and the leading valence electronic configuration.

In the case of the transition towards the LUMO, we consider only the dominant decay pathways which represent about 
$90 \%$ of the channels. It is seen in Table 1 that spectator Auger decay channels, for which the excited electron does not participate in the decay (i.e. the excited electron remains in the $1 b_{1}$ after the decay), are the dominant ones. Participator Auger decay represents only about $10 \%$ of the overall process. Similarly, spectator Auger decay channels are dominant after the transition from the $1 \mathrm{~s}$ level of carbon to the singly occupied HOMO, as seen in Table 2.

The Auger decay of both core-excited states leads to a larger population in the singlet states than in the triplet states. This was already observed in several molecules ${ }^{28}$. In each spin symmetry, the states exhibit populations of similar magnitude. Furthermore, the branching ratios for shake-up Auger processes (not shown), in which the photoexcited electron is further excited to a higher lying orbital during the Auger process, are negligible for this system.

\begin{tabular}{llcccc}
\hline & & Auger & $\mathrm{CH}_{2}^{2+}$ & $\mathrm{CH}^{+} / \mathrm{H}^{+}$ & $\mathrm{C}^{+} / \mathrm{H}^{+} / \mathrm{H}$ \\
\hline${ }^{1} \mathrm{~A}_{1}$ & $\left(2 \mathrm{a}_{1}\right)^{2}\left(1 \mathrm{~b}_{2}\right)^{2}$ & 10 & 100 & 0 & 0 \\
${ }^{1} \mathrm{~A}_{2}$ & $\left(2 \mathrm{a}_{1}\right)^{2}\left(1 \mathrm{~b}_{2}\right)^{1}\left(1 \mathrm{~b}_{1}\right)^{1}$ & 9 & 0 & 32 & 68 \\
${ }^{1} \mathrm{~B}_{1}$ & $\left(2 \mathrm{a}_{1}\right)^{2}\left(3 \mathrm{a}_{1}\right)^{1}\left(1 \mathrm{~b}_{1}\right)^{1}$ & 7 & 0 & 0 & 100 \\
${ }^{1} \mathrm{~B}_{2}$ & $\left(2 \mathrm{a}_{1}\right)^{1}\left(1 \mathrm{~b}_{2}\right)^{2}\left(1 \mathrm{~b}_{1}\right)^{1}$ & 15 & 85 & 14 & 1 \\
${ }^{1} \mathrm{~B}_{1}$ & $\left(1 \mathrm{~b}_{2}\right)^{2}\left(3 \mathrm{a}_{1}\right)^{1}\left(1 \mathrm{~b}_{1}\right)^{1}$ & 9 & 0 & 0 & 100 \\
${ }^{3} \mathrm{~A}_{2}$ & $\left(2 \mathrm{a}_{1}\right)^{2}\left(1 \mathrm{~b}_{2}\right)^{1}\left(1 \mathrm{~b}_{1}\right)^{1}$ & 9 & 0 & 89 & 11 \\
${ }^{3} \mathrm{~B}_{1}$ & $\left(2 \mathrm{a}_{1}\right)^{2}\left(3 \mathrm{a}_{1}\right)^{1}\left(1 \mathrm{~b}_{1}\right)^{1}$ & 15 & 0 & 21 & 79 \\
${ }^{3} \mathrm{~B}_{1}$ & $\left(1 \mathrm{~b}_{2}\right)^{2}\left(3 \mathrm{a}_{1}\right)^{1}\left(1 \mathrm{~b}_{1}\right)^{1}$ & 17 & 0 & 0 & 100 \\
\hline
\end{tabular}

Table 1 Auger and fragmentation patterns branching ratios (in \%) after resonant Auger in $\mathrm{CH}_{2}^{+}$for the transition $1 a_{1} \rightarrow 1 b_{1}$. The final Auger states are shown in increasing energy order from top to bottom (the energies of the dicationic states at the ground state equilibrium geometry are given in Supporting information). Final states with smaller Auger branching ratio were neglected. The sum of their contribution corresponds to the remaining $9 \%$.

Subsequently, we simulated the fragmentation dynamics of each of the final Auger states. For each of these doublyionized states, we ran 100 trajectories starting on the corresponding potential energy surface. For each trajectory, we computed the size of the fragments containing the carbon atom after reaching the asymptotic distances (after $150 \mathrm{fs}$ ). The branching ratios of each fragment are given in Table 1 and Table 2 for all final states. As seen in both tables, after Auger decay to the lowest state of $\mathrm{CH}_{2}^{2+}$, the system remains bound. Indeed, the lowest state of $\mathrm{CH}_{2}^{2+}$ is stable and has an equilibrium geometry close to that of $\mathrm{CH}_{2}^{+}$which explains the near unity branching ratio of the $\mathrm{CH}_{2}^{2+}$ fragment. For higher states, $\mathrm{CH}_{2}^{2+}$ dissociates into either $\mathrm{CH}^{+}$and $\mathrm{H}^{+}$or $\mathrm{C}^{+}, \mathrm{H}^{+}$and a neutral $\mathrm{H}$, respectively. For both transitions (i.e. towards the LUMO and HOMO), the total branching ratio for the channel $\mathrm{C}^{+} / \mathrm{H}^{+} / \mathrm{H}$ dominates that of $\mathrm{CH}^{+} / \mathrm{H}^{+}$.

These fragments can further react with electrons, ions, atoms and molecules present in the ISM. In order to provide a complete description of the x-ray induced chemistry, the corresponding reaction rates are needed ${ }^{29-33}$. The latter depend strongly on the collision energy and thus on the kinetic energies of the fragments. Furthermore, the reaction rates may be different if the fragments are not "cold". We therefore provide here the kinetic and internal energy distributions of the fragments containing the carbon atom after the fragmentation is complete.

The kinetic energy distributions of $\mathrm{CH}^{+}$and $\mathrm{C}^{+}$fragments are shown in Fig. 3 for both core-excited states. These distributions are obtained by weighting the distribution computed for each final state with the corresponding Auger branching ratio. Since $\mathrm{CH}_{2}^{2+}$ does not dissociate it gains no kinetic energy after the Auger decay and it is not shown here. For the smaller fragments, another proton is formed during the dissociation. Owing to the Coulomb repulsion between the proton and the remaining ionic fragments, the latter acquire kinetic energy of up to $1 \mathrm{eV}$, during the fragmentation. 


\begin{tabular}{llcccc}
\hline & Auger & $\mathrm{CH}_{2}^{2+}$ & $\mathrm{CH}^{+} / \mathrm{H}^{+}$ & $\mathrm{C}^{+} / \mathrm{H}^{+} / \mathrm{H}$ \\
\hline${ }^{1} \mathrm{~A}_{1}$ & $\left(2 \mathrm{a}_{1}\right)^{2}\left(1 \mathrm{~b}_{2}\right)^{2}$ & 17 & 99 & 1 & 0 \\
${ }^{1} \mathrm{~B}_{2}$ & $\left(2 \mathrm{a}_{1}\right)^{2}\left(1 \mathrm{~b}_{2}\right)^{1}\left(3 \mathrm{a}_{1}\right)^{1}$ & 11 & 0 & 63 & 37 \\
${ }^{1} \mathrm{~A}_{1}$ & $\left(2 \mathrm{a}_{1}\right)^{2}\left(3 \mathrm{a}_{1}\right)^{2}$ & 16 & 4 & 8 & 88 \\
${ }^{1} \mathrm{~A}_{1}$ & $\left(2 \mathrm{a}_{1}\right)^{1}\left(1 \mathrm{~b}_{2}\right)^{2}\left(3 \mathrm{a}_{1}\right)^{1}$ & 15 & 50 & 41 & 9 \\
${ }^{1} \mathrm{~B}_{2}$ & $\left(2 \mathrm{a}_{1}\right)^{1}\left(1 \mathrm{~b}_{2}\right)^{1}\left(3 \mathrm{a}_{1}\right)^{2}$ & 11 & 0 & 0 & 100 \\
${ }^{1} \mathrm{~A}_{1}$ & $\left(1 \mathrm{~b}_{2}\right)^{2}\left(3 \mathrm{a}_{1}\right)^{2}$ & 13 & 0 & 0 & 100 \\
${ }^{3} \mathrm{~B}_{2}$ & $\left(2 \mathrm{a}_{1}\right)^{2}\left(1 \mathrm{~b}_{2}\right)^{1}\left(3 \mathrm{a}_{1}\right)^{1}$ & 5 & 0 & 80 & 20 \\
${ }^{3} \mathrm{~A}_{1}$ & $\left(2 \mathrm{a}_{1}\right)^{1}\left(1 \mathrm{~b}_{2}\right)^{2}\left(3 \mathrm{a}_{1}\right)^{1}$ & 7 & 32 & 66 & 2 \\
${ }^{3} \mathrm{~B}_{2}$ & $\left(2 \mathrm{a}_{1}\right)^{1}\left(1 \mathrm{~b}_{2}\right)^{1}\left(3 \mathrm{a}_{1}\right)^{2}$ & 5 & 0 & 1 & 99 \\
\hline
\end{tabular}

Table 2 Auger and fragmentation patterns branching ratios (in \%) after resonant Auger in $\mathrm{CH}_{2}^{+}$for the transition $1 a_{1} \rightarrow 3 a_{1}$. The final Auger states are shown in increasing energy order from top to bottom (the energies of the dicationic states at the ground state equilibrium geometry are given in Supporting information).

The internal energy distributions of $\mathrm{CH}_{2}^{2+}$ and $\mathrm{CH}^{+}$fragments are shown in Fig. 4. These distributions are calculated

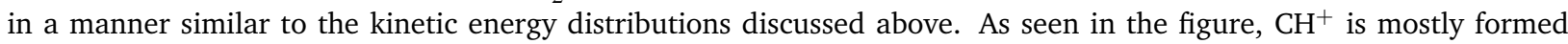
with low internal energy, but the distribution extends up to $2 \mathrm{eV}$. The stretching frequency of $\mathrm{CH}^{+}$corresponds to a vibrational energy spacing of about $0.3 \mathrm{eV}$. In a quantum description, $\mathrm{CH}^{+}$ions are therefore formed in vibrational states up to $v=6$.

The internal energy distributions of $\mathrm{CH}_{2}^{2+}$ extend from $0.0 \mathrm{eV}$ to about $1.0 \mathrm{eV}$. It should be noted that the different modes (symmetric and antisymmetric stretching and bending) of $\mathrm{CH}_{2}^{2+}$ are not distinguished here.

As remarked above, the dominant decay channel following x-ray excitation of $\mathrm{CH}^{+}$is $\mathrm{C}^{2+} / \mathrm{H}$ (see $\mathrm{Ref}^{9}$ ). For $\mathrm{CH}_{2}^{+}$ we estimate the decay channel $\mathrm{C}^{2+} / \mathrm{H} / \mathrm{H}$ to be only $3 \%$ of the overall products and so it was neglected in the above discussion. However, a recent merged beam photo absorption experiment has measured this particular channel: $\mathrm{CH}_{2}^{+}$ ions produced in an ECR ion source were probed with x-ray photons ranging from 280-310 eV. For each photon energy, the $\mathrm{C}^{2+}$ fragment counting rate was measured which provides the cross section for this channel (see Supporting information for details).This experiment confirms that the decay channel $\mathrm{C}^{2+} / \mathrm{H} / \mathrm{H}$ is indeed weak since the corresponding cross sections are below $1 \mathrm{Mb}$ for all transitions. Furthermore, as shown in Supporting information, there is a reasonable quantitative agreement between experiment and theory, even for such a weak channel.

$\mathrm{CH}_{3}^{+}$

The ground state equilibrium geometry of $\mathrm{CH}_{3}^{+}$is planar and has a $\mathrm{D}_{3 h}$ symmetry. Its electronic configuration is $\left(1 \mathrm{a}_{1}^{\prime}\right)^{2}\left(2 \mathrm{a}_{1}^{\prime}\right)^{2}\left(\mathrm{e}^{\prime}\right)^{4}$. The $1 \mathrm{a}_{1}^{\prime}$ orbital corresponds to the 1 s orbital of carbon.

The x-ray absorption spectrum of $\mathrm{CH}_{3}^{+}$is shown in Fig. 5. The core-shell ionization threshold was computed at 306.6 $\mathrm{eV}$. The most intense band, centered below $285 \mathrm{eV}$, exhibits a large vibrational progression and corresponds to a single electronic transition from the 1 s orbital of carbon to the lowest unoccupied molecular orbital (i.e. to $1 a_{2}^{\prime \prime}$ orbital). Higher lying transitions correspond to excitation towards higher unoccupied orbitals. Below we focus on the decay and fragmentation processes following the lowest in energy and most intense core excitation.

In contrast to $\mathrm{CH}_{2}^{+}$, only doublet states are accessible after resonant Auger decay in $\mathrm{CH}_{3}^{+}$. The branching ratios for the different channels are shown in Table 3. As seen in the table, the most populated states correspond to spectator Auger 
decay, in which the excited electron remains in the $1 \mathrm{a}_{2}^{\prime \prime}$ orbital. These channels represent more than $60 \%$ of the total decay.

\begin{tabular}{ccccccc}
\hline & & Auger & $\mathrm{CH}_{3}^{2+}$ & $\mathrm{CH}_{2}^{+} / \mathrm{H}^{+}$ & $\mathrm{CH}^{+} / \mathrm{H}^{+} / \mathrm{H}$ & $\mathrm{C}^{+} / \mathrm{H}^{+} / \mathrm{H} / \mathrm{H}$ \\
\hline${ }^{2} \mathrm{E}^{\prime}$ & $\left(2 \mathrm{a}_{1}^{\prime}\right)^{2}\left(\mathrm{e}^{\prime}\right)^{3}$ & 13 & 1 & 99 & 0 & 0 \\
${ }^{2} \mathrm{~A}_{2}^{\prime \prime}$ & $\left(2 \mathrm{a}_{1}^{\prime}\right)^{2}\left(\mathrm{e}^{\prime}\right)^{2}\left(1 \mathrm{a}_{2}^{\prime \prime}\right)^{1}$ & 7 & 1 & 83 & 16 & 0 \\
${ }^{2} \mathrm{E}^{\prime}$ & $\left(2 \mathrm{a}_{1}^{\prime}\right)^{2}\left(\mathrm{e}^{\prime}\right)^{2}\left(1 \mathrm{a}_{2}^{\prime \prime}\right)^{1}$ & 14 & 4 & 85 & 11 & 0 \\
${ }^{2} \mathrm{~A}_{2}^{\prime \prime}$ & $\left(2 \mathrm{a}_{1}^{\prime}\right)^{2}\left(\mathrm{e}^{\prime}\right)^{2}\left(1 \mathrm{a}_{2}^{\prime \prime}\right)^{1}$ & 8 & 8 & 65 & 25 & 0 \\
${ }^{2} \mathrm{~A}_{1}^{\prime}$ & $\left(2 \mathrm{a}_{1}^{\prime}\right)^{1}\left(\mathrm{e}^{\prime}\right)^{4}$ & 4 & 71 & 22 & 7 & 0 \\
${ }^{2} \mathrm{E}^{\prime}$ & $\left(2 \mathrm{a}_{1}^{\prime}\right)^{1}\left(\mathrm{e}^{\prime}\right)^{3}\left(1 \mathrm{a}_{2}^{\prime \prime}\right)^{1}$ & 23 & 4 & 63 & 23 & 10 \\
${ }^{2} \mathrm{E}^{\prime}$ & $\left(2 \mathrm{a}_{1}^{\prime}\right)^{1}\left(\mathrm{e}^{\prime}\right)^{3}\left(1 \mathrm{a}_{2}^{\prime \prime}\right)^{1}$ & 6 & 3 & 54 & 41 & 2 \\
${ }^{2} \mathrm{~A}_{2}^{\prime \prime}$ & $\left(\mathrm{e}^{\prime}\right)^{4}\left(1 \mathrm{a}_{2}^{\prime \prime}\right)^{1}$ & 12 & 0 & 76 & 23 & 1 \\
\hline
\end{tabular}

Table 3 Auger and fragmentation patterns branching ratios (in \%) after resonant Auger in $\mathrm{CH}_{3}^{+}$for the transition $1 \mathrm{a}_{1}^{\prime} \rightarrow 1 \mathrm{a}_{2}^{\prime \prime}$. The final Auger states are shown in increasing energy order from top to bottom (the energies of the dicationic states at the ground state equilibrium geometry are given in Supporting information).

The nuclear dynamics following the Auger decay was simulated for each channel with 100 trajectories. The branching ratios of the different fragmentations for each state are shown in Table 3. Except for the ${ }^{2} \mathrm{~A}_{1}^{\prime}$ state which is weakly populated by the resonant Auger decay, the molecular ion $\mathrm{CH}_{3}^{+}$breaks dominantly into $\mathrm{CH}_{2}^{+}$. In the commonly accepted model of the formation of $\mathrm{CH}_{2}^{+}$and $\mathrm{CH}_{3}^{+}$, the former is produced from atomic carbon ions and the latter from $\mathrm{CH}_{2}^{+}$. However, the results shown here demonstrate that in the presence of a soft x-ray source $\mathrm{CH}_{3}^{+}$is transformed back into $\mathrm{CH}_{2}^{+}$.

As explained for $\mathrm{CH}_{2}^{+}$, the kinetic and internal energy distributions of the fragments are essential input data for the modeling of subsequent reactions of these species. As shown in Fig. 6, all fragments containing the carbon atom have kinetic energy below $0.7 \mathrm{eV}$. The internal energy distributions of the fragments are shown in Fig. 7. The distribution of the $\mathrm{CH}_{3}^{2+}$ is nearly zero above $0.8 \mathrm{eV}$. In contrast, $\mathrm{CH}_{2}^{+}$and $\mathrm{CH}^{+}$fragments have internal energies extending up to $2 \mathrm{eV}$, showing that these species are vibrationally excited after the fragmentation of $\mathrm{CH}_{3}^{2+}$.

\section{Conclusions}

In conclusion, we used a combination of electronic structure calculations, resonant scattering approaches and molecular dynamics techniques to provide a complete description of the x-ray induced chemistry of $\mathrm{CH}_{2}^{+}$and $\mathrm{CH}_{3}^{+}$.

We showed that these ions exhibit strong x-ray absorption lines. After core-excitation, these systems relax through Auger decay within $7 \mathrm{fs}$. The doubly-charged ions thus formed mostly dissociate into smaller ionic carbon fragments: in the case of $\mathrm{CH}_{2}^{+}$, the dominant products are either $\mathrm{C}^{+} / \mathrm{H}^{+} / \mathrm{H}$ or $\mathrm{CH}^{+} / \mathrm{H}^{+}$. For $\mathrm{CH}_{3}^{+}$, the system breaks primary into $\mathrm{CH}_{2}^{+}$ and $\mathrm{H}^{+}$. The latter case shows a new route to form $\mathrm{CH}_{2}^{+}$near an x-ray source. Furthermore, we reported the kinetic and internal energy distributions of these product fragments. They all have low $(<1 \mathrm{eV})$ kinetic energy after fragmentation and are in general vibrationally excited.

The two molecular ions $\mathrm{CH}_{2}^{+}$and $\mathrm{CH}_{3}^{+}$are important intermediate species in the formation of complex molecules in the interstellar medium. The quantitative determination of the products formed upon x-ray irradiation as well as their kinetic and internal energy distributions should help to set up a more complete description of the chemical reactions in interstellar regions near x-ray sources.

\section{Acknowledgement}

This project has received funding from the Research Executive Agency (REA) under the European Union's Horizon 2020 research and innovation programme Grant agreement No 705515 and from the LABEX Plas@par (ANR-11-IDEX-0004- 
02). E.T.K, J-P.M., J-M.B. and D.C. wish to thank the PLEIADES beam staff, for help during the experiments, and the European Commission (FP7) for financial assistance.

\section{References}

1 D. W. Savin, N. S. Brickhouse, J. J. Cowan, R. P. Drake, S. R. Federman, G. J. Ferland, A. Frank, M. S. Gudipati, W. C. Haxton, E. Herbst, S. Profumo, F. Salama, L. M. Ziurys and E. G. Zweibel, Reports on Progress in Physics, 2012, 75, 036901.

2 A. G. G. M. Tielens, Rev. Mod. Phys., 2013, 85, 1021-1081.

3 http://kida.obs.u-bordeauxl.fr.

4 V. Wakelam, J.-C. Loison, E. Herbst, B. Pavone, A. Bergeat, K. Béroff, M. Chabot, A. Faure, D. Galli, W. D. Geppert, D. Gerlich, P. Gratier, N. Harada, K. M. Hickson, P. Honvault, S. J. Klippenstein, S. D. L. Picard, G. Nyman, M. Ruaud, S. Schlemmer, I. R. Sims, D. Talbi, J. Tennyson and R. Wester, The Astrophysical Journal Supplement Series, 2015, 217, 20 .

5 http://www.udfa.net/.

6 E. F. van Dishoeck, B. Jonkheid and M. C. van Hemert, Faraday Discuss., 2006, 133, 231-243.

7 T. J. Millar, Plasma Sources Science and Technology, 2015, 24, 043001.

8 J. Greiner, New Astronomy, 2000, 5, 137 - 141.

9 J.-P. Mosnier, E. T. Kennedy, P. van Kampen, D. Cubaynes, S. Guilbaud, N. Sisourat, A. Puglisi, S. Carniato and J.-M. Bizau, Phys. Rev. A, 2016, 93, 061401.

10 T. D. Thomas, L. J. Saethre, S. L. Sorensen and S. Svensson, J. Chem. Phys., 1998, 109, 1041-1051.

11 M. W. Schmidt, K. K. Baldridge, J. A. Boatz, S. T. Elbert, M. S. Gordon, J. H. Jensen, S. Koseki, N. Matsunaga, K. A. Nguyen, S. Su, T. L. Windus, M. Dupuis and J. A. Montgomery, 1993, 14, 1347-1363.

12 A. Puglisi, N. Sisourat and S. Carniato, AIP Conference Proceedings, 2017, 1811, 130002.

13 T. H. Dunning, J. Chem. Phys., 1989, 90, 1007-1023.

14 C. Nicolas and C. Miron, J. Electron Spectrosc. Relat. Phenom., 2012, 185, 267 - 272.

15 T. Miteva, S. Kazandjian and N. Sisourat, Chem. Phys., 2017, 482, 208.

16 U. Fano, Phys. Rev., 1961, 124, 1866-1878.

17 G. Howat, T. Åberg and O. Goscinski, J. Phys. B At. Mol. Opt. Phys., 1978, 11, 1575-1588.

18 A. U. Hazi, Electron-Molecule and Photon-Molecule Collisions, Plenum Press, New York and London, 1979.

19 P. W. Langhoff, Electron-Molecule and Photon-Molecule Collisions, Plenum Press, New York and London, 1979.

20 F. Müller-Plathe and G. H. F. Diercksen, Phys. Rev. A, 1989, 40, 696-711.

21 I. Cherkes, S. Klaiman and N. Moiseyev, International Journal of Quantum Chemistry, 2009, 109, $2996-3002$. 
22 M. Richter, P. Marquetand, J. González-Vázquez, I. Sola and L. González, J. Chem. Theory Comput., 2011, 7, 12531258.

23 S. Mai, P. Marquetand and L. González, Int. J. Quant. Chem., 2015, 115, 1215-1231.

24 H.-J. Werner, P. J. Knowles, G. Knizia, F. R. Manby, M. Schütz, P. Celani, W. Györffy, D. Kats, T. Korona, R. Lindh, A. Mitrushenkov, G. Rauhut, K. R. Shamasundar, T. B. Adler, R. D. Amos, A. Bernhardsson, A. Berning, D. L. Cooper, M. J. O. Deegan, A. J. Dobbyn, F. Eckert, E. Goll, C. Hampel, A. Hesselmann, G. Hetzer, T. Hrenar, G. Jansen, C. Köppl, Y. Liu, A. W. Lloyd, R. A. Mata, A. J. May, S. J. McNicholas, W. Meyer, M. E. Mura, A. Nicklass, D. P. O’Neill, P. Palmieri, D. Peng, K. Pflüger, R. Pitzer, M. Reiher, T. Shiozaki, H. Stoll, A. J. Stone, R. Tarroni, T. Thorsteinsson and M. Wang, MOLPRO, version 2015.1, a package of ab initio programs.

25 H.-J. Werner, P. J. Knowles, G. Knizia, F. R. Manby and M. Schütz, WIREs Comput Mol Sci, 2012, 2, $242-253$.

26 R. Ditchfield, W. J. Hehre and J. A. Pople, The Journal of Chemical Physics, 1971, 54, 724-728.

27 G. Theodorakopoulos and I. D. Petsalakis, Journal of Molecular Structure: THEOCHEM, 1991, 230, 205 - 211.

28 F. Tarantelli, A. Sgamellotti, L. S. Cederbaum and J. Schirmer, J. Chem. Phys., 1987, 86, 2201-2206.

29 R. K. Janev and D. Reiter, Physics of Plasmas, 2002, 9, 4071-4081.

30 R. D. Thomas, Mass Spectrometry Reviews, 2008, 27, 485-530.

31 J. Lecointre, D. S. Belic, J. J. Jureta, R. K. Janev and P. Defrance, Eur. Phys. J. D, 2009, 55, 569.

32 J. Lecointre, D. S. Belic, J. J. Jureta, R. K. Janev and P. Defrance, Eur. Phys. J. D, 2009, 55, 557.

33 M. Fogle, E. M. Bahati, M. E. Bannister, S. H. M. Deng, C. R. Vane, R. D. Thomas and V. Zhaunerchyk, Phys. Rev. A, 2010, 82, 042720. 

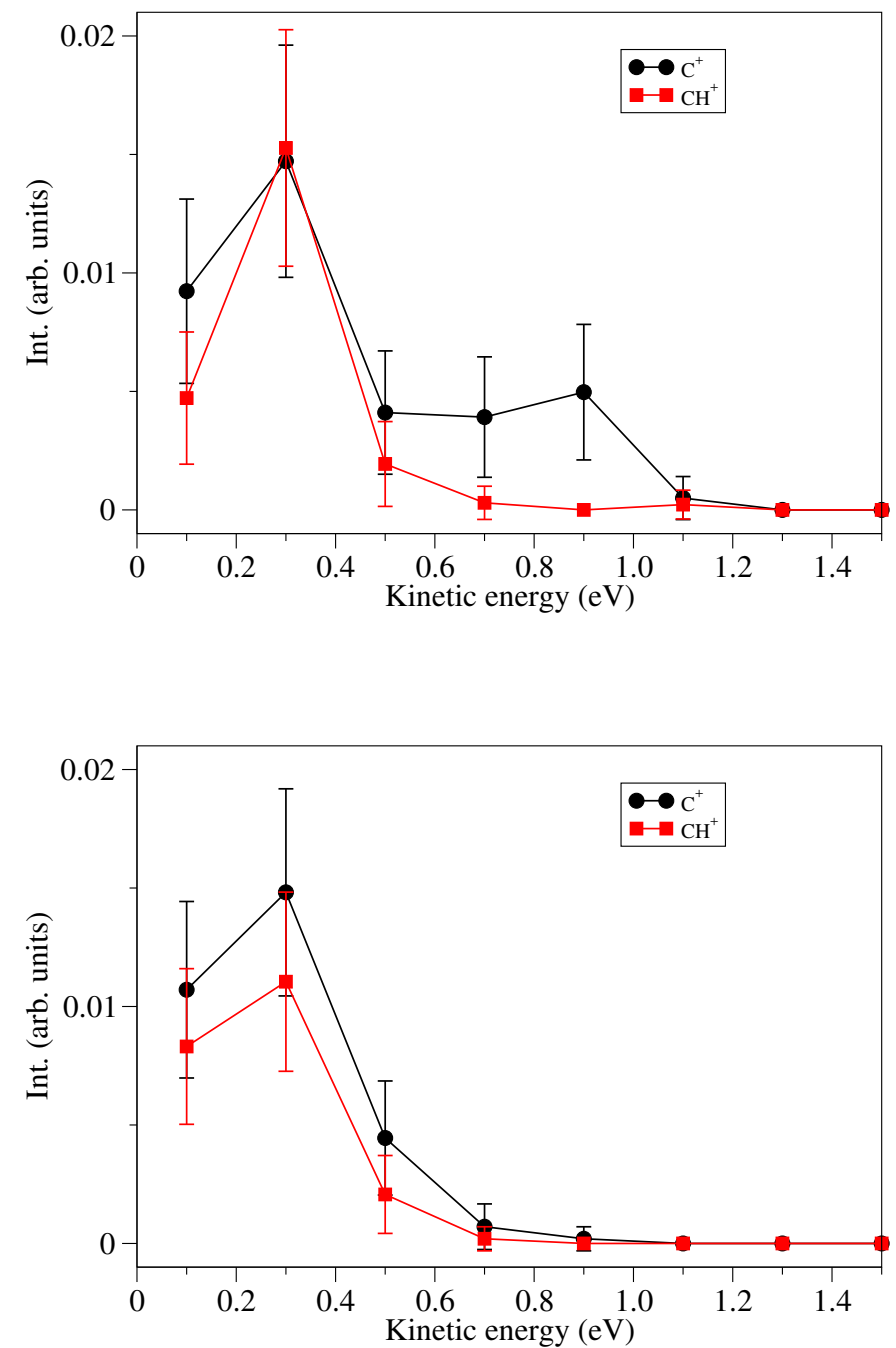

Fig. 3 Kinetic energy distribution of the fragments containing the carbon atom, following the resonant Auger decay in $\mathrm{CH}_{2}^{+}$. Error bars correspond to the statistical error due to the finite number of trajectories. Top panel: for the transition $1 a_{1} \rightarrow 1 b_{1}$ (LUMO). Bottom panel: for the transition $1 a_{1} \rightarrow 3 a_{1}$ (HOMO). 

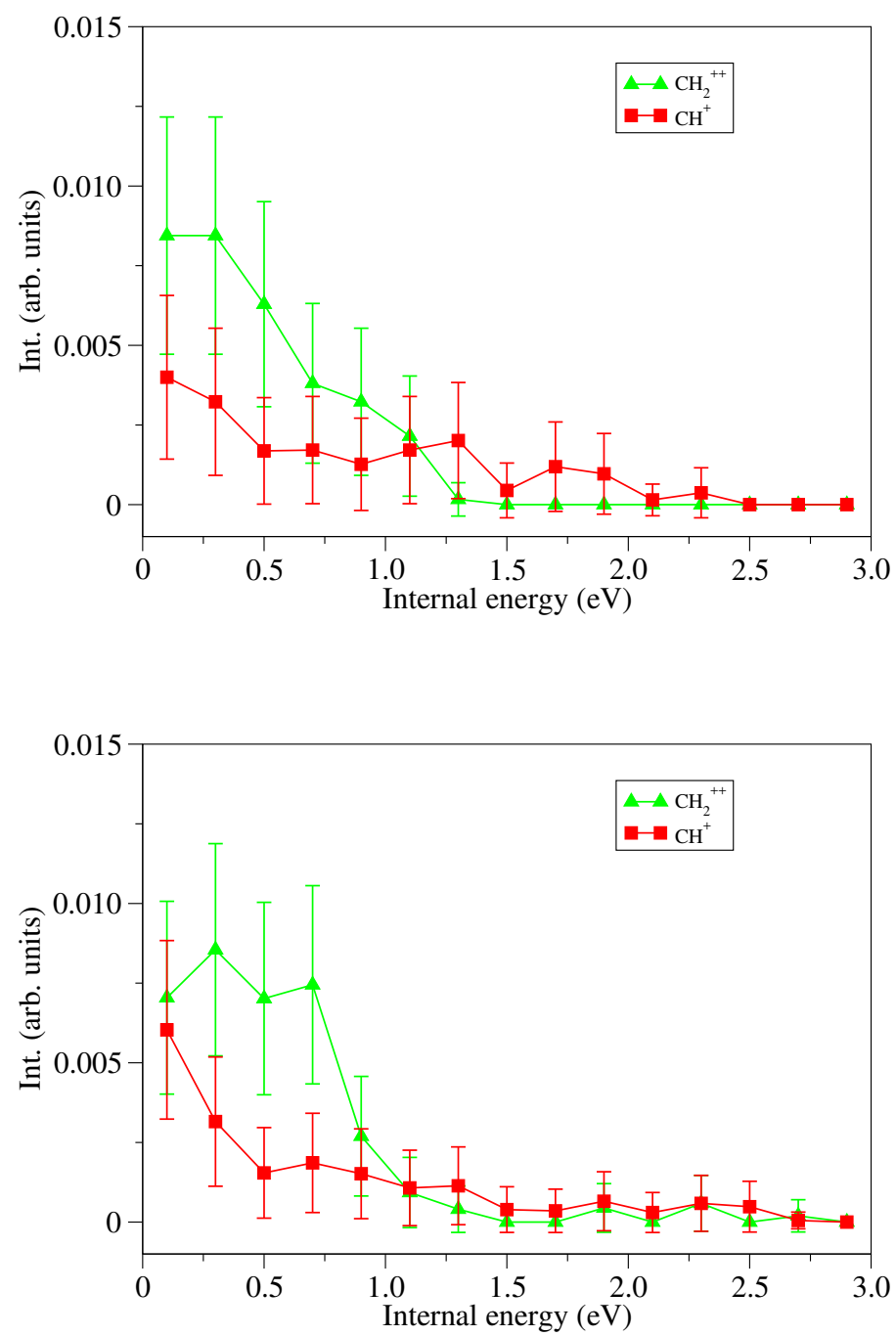

Fig. 4 Internal energy distribution of the fragments containing the carbon atom, following the resonant Auger decay in $\mathrm{CH}_{2}^{+}$. Error bars correspond to the statistical error due to the finite number of trajectories. Top panel: for the transition $1 a_{1} \rightarrow 1 b_{1}$ (LUMO). Bottom panel: for the transition $1 a_{1} \rightarrow 3 a_{1}$ (HOMO). 


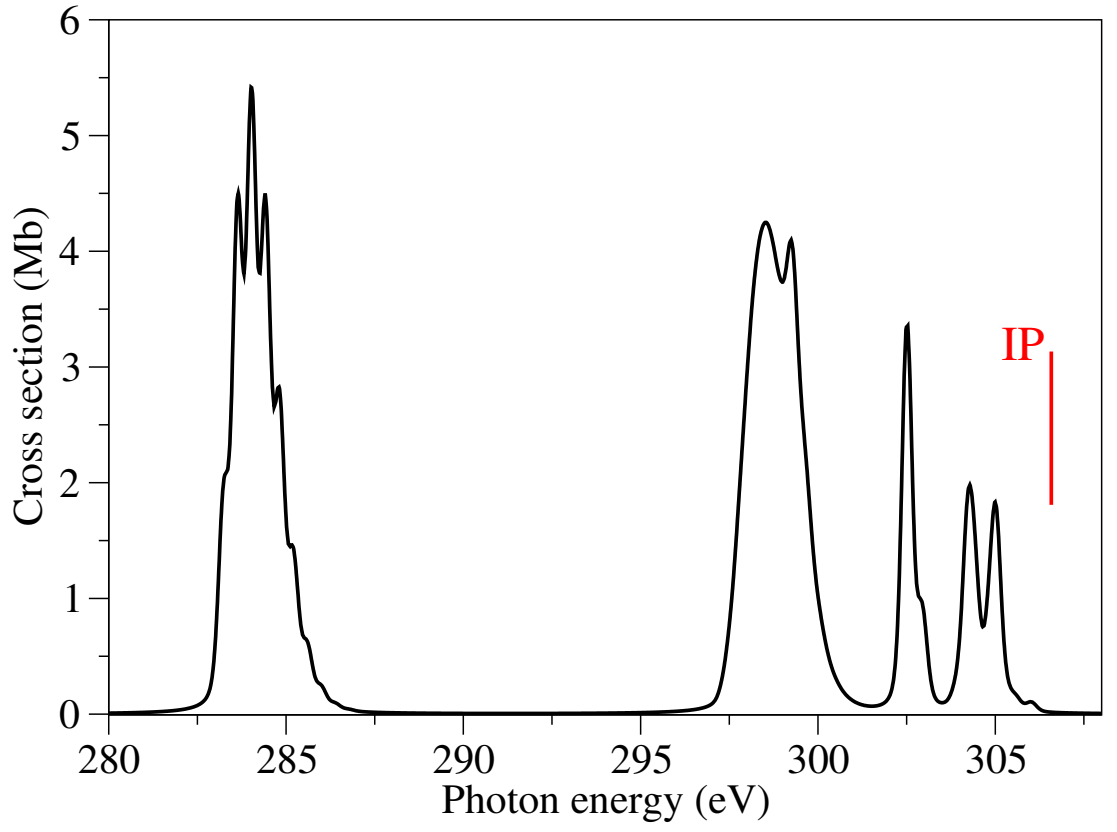

Fig. 5 Absolute x-ray photoabsorption cross sections of $\mathrm{CH}_{3}^{+}$, calculated using the linear coupling model (see Eq. 1). 


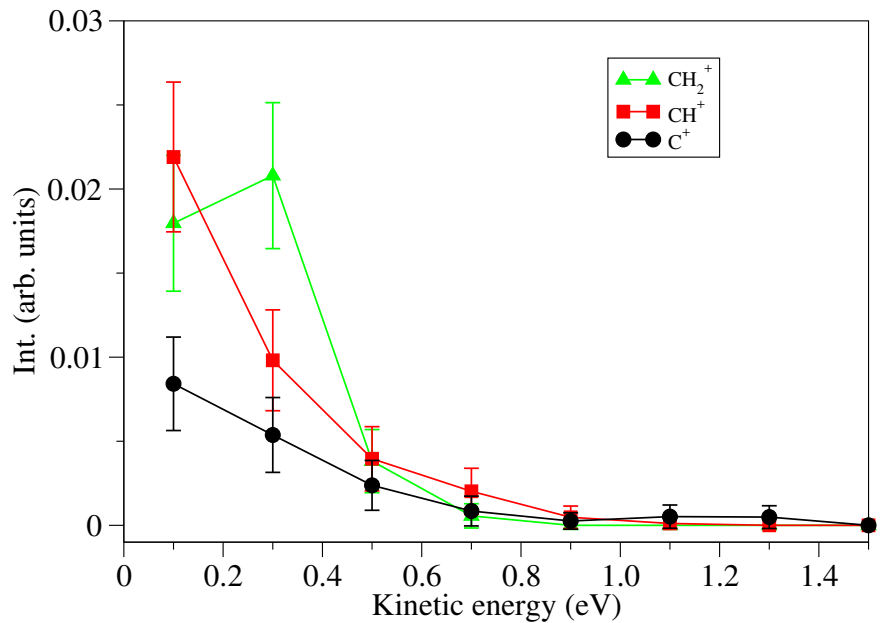

Fig. 6 Kinetic energy distribution of the fragments containing the carbon atom, following the resonant Auger decay in $\mathrm{CH}_{3}^{+}$for the transition $1 \mathrm{a}_{1}^{\prime} \rightarrow 1 \mathrm{a}_{2}^{\prime \prime}$. Error bars correspond to the statistical error due to the finite number of trajectories.

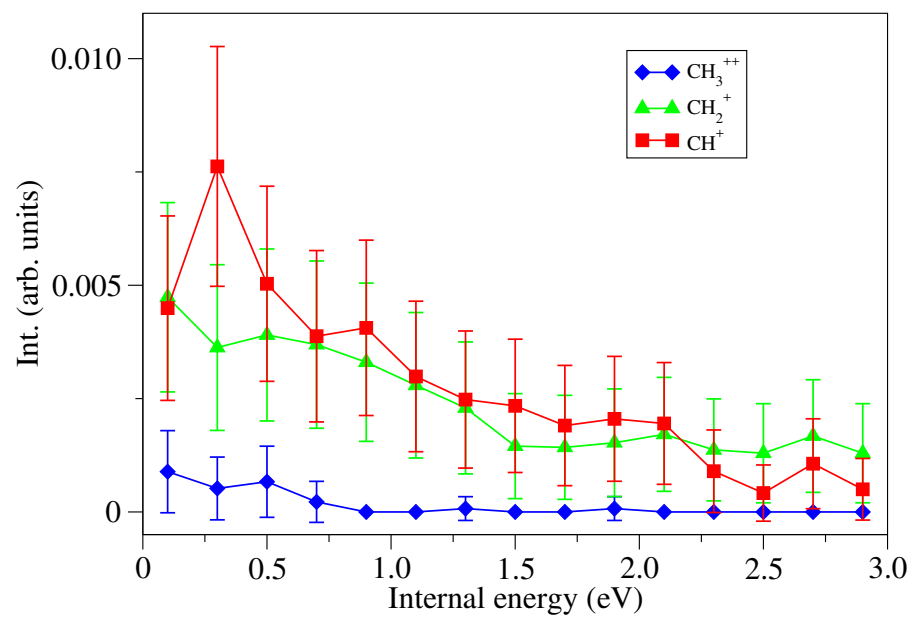

Fig. 7 Internal energy distribution of the fragments containing the carbon atom, following the resonant Auger decay in $\mathrm{CH}_{3}^{+}$for the transition $1 \mathrm{a}_{1}^{\prime} \rightarrow 1 \mathrm{a}_{2}^{\prime \prime}$. Error bars correspond to the statistical error due to the finite number of trajectories. 